BNL-73359-2004-CP

\title{
The BNL Super Neutrino Beam Project
}

\author{
W-T. Weng and D. Raparia \\ (on behalf of the BNL Neutrino Working Group) \\ Presented at $33^{\text {rd }}$ Advanced Beam Dynamics Workshop (ICFA-HB2004) \\ Bensheim, Germany \\ October 18-22; 2004
}

\author{
Center for Accelerator Physics \\ Brookhaven National Laboratory \\ P.O. Box 5000 \\ Upton, NY 11973-5000 \\ www:bnl:gov \\ Managed by \\ Brookhaven Science Associates, LLC \\ for the United States Department of Energy under \\ Contract No. DE-AC02-98CH10886
}

This is a preprint of a paper intended for publication in a journal or proceedings. Since changes may be made before publication, this preprint is made available with the understanding that it will not be cited or reproduced without the permission of the author. 


\section{DISCLAIMER}

This report was prepared as an account of work sponsored by an agency of the United States Government: Neither the United States Government nor any agency thereof, nor any of their employees, nor any of their contractors; subcontractors or their employees, makès any warranty; express or implied, or assumes any legal liability or responsibility for the accuracy, completeness, or any third party's use or the results of such use of any information, apparatus; product; or process disclosed, or represents that its use would not infringe privately owned rights. Reference herein to any specific commercial product, process, or service by trade name, trademark, manufacturer, or otherwise, doès not necessarily constitute or imply its endorsement;: recommendation, : or favoring by the United States Government or any agency thereof or its contractors or subcontractors: The views and opinions of authors expressed herein do not necessarily state or reflect those of the United States Government or any agency thereof. 


\title{
THE BNL SUPER NEUTRINO BEAM PROJECT*
}

\author{
W-T. Weng*** and D. Raparia: \\ (On Behalf of the BNL Neutrino.Working Group) \\ Brookhaven National Laboratory, Upton, NY 11973,USA
}

\section{Abstract.}

To determine the neutrino mixing amplitudes and phase. accurately, as well as the $\mathrm{CP}$. violation parameters; a very: long base line super neutrino beam facility is needed. This is possible due to the long distance and wideband nature of: the neutrino beam for the observation of several oscillations : from one species : of the neutrino to the other: $[1,2]$

BNL plans to upgrade the AGS proton beam from the current $0.14 \mathrm{MW}$ to higher than 1.0 MW and beyond for:.. such a neutrino facility which consists of three major subsystems. First is a $1.5 \mathrm{GeV}$ superconducting linac to replace the booster as injector for the AGS; second is the performance upgrade for the AGS itself: for the higher intensity and repetition rate, and finally is target and horn system for the neutrino .... production.. The major contribution for the higher power is from the increase of the repetition rate of the AGS form $0.3 \mathrm{~Hz}$ to $2.5 \mathrm{~Hz}$, with. moderate increase from the intensity [3]

The design consideration to achieve high intensity and low losses for the linac and the AGS will be reviewed. The target horn design for high power operation and easy .. maintenance will also be presented.

\section{INTRODUCTION}

We have examined possible upgrades to the AGS complex that would meet the requirements of the proton beam for a 1.0 MW neutrino superbeam facility: We are proposing to replace part of the existing $200 \mathrm{MeV}$ linac with coupled cavity structure from $116 \mathrm{MeV}$ to $400 \mathrm{MeV}$. and then add additional $1.1 \mathrm{GeV}$ superconducting linac to reach a final energy of $1.5 \mathrm{GeV}$ for direct $\mathrm{H}^{-}$injection into the AGS.

The requirements of the proton beam for the super neutrino beam are summarized in Table 1 and a layout of upgraded AGS is shown in Figure 1 . Since the present: number of protons per fill is already close to the required number, the upgrade focuses on increasing the repetition. rate and reducing beam losses (to avoid excessive shielding requirements and to maintain activation of the machine : components at workable level). It is also important to preserve all the present capabilities of the AGS, in particular its role as injector to RHIC.

Present injection $\cdots$ into $:$ the AGS requires the accumulation of four Booster loads in the AGS, which takes about $0.6 \mathrm{sec}$, and is therefore not suited for high average beam power operation.
*Work performed under the auspices of the US Department of Energy
**E-mail weng@bnl.gov.
TABLE 1. AGS Proton Driver Parameters.

\begin{tabular}{ll}
\hline Total beam power & $1 \mathrm{MW}$ \\
Beam energy ... & $28 \cdot \mathrm{GeV}$ \\
Average beam current & $42 \mu \mathrm{A}$ \\
Cycle time & $400 \mathrm{msec}$ \\
Number of protons per fill & $0.9 \times 10^{14} \cdot$ \\
Number of bunches per fill & 24 \\
Protons per bunch & $0.4 \times 10^{13}$ \\
Injection turns & 230 \\
Repetition rate & $2.5 \cdot \mathrm{Hz}$ \\
Pulse length & $0.72 \mathrm{msec}$ \\
Chopping rate & 0.75 \\
Linac average/peak current & $20 / 30 \mathrm{~mA}$ \\
\hline
\end{tabular}

To minimize the injection time to about $1 \mathrm{msec}$, a 1.5 $\mathrm{GeV}$ linac will be used instead. The multi-turn injection from: a source of $28 \mathrm{~mA}$ and $720 \mu \mathrm{sec}$ pulse width is sufficient to accumulate $0.9 \times 10^{14}$ particle per pulse in the AGS. The minimum ramp time of the AGS to full energy: is presently: $0.5 \mathrm{sec}$. This must be reduced down to 0.2 sec to reach the required repetition rate of $2.5 \mathrm{~Hz}$ to deliver the required $1 \mathrm{MW}$ beam to the garget.

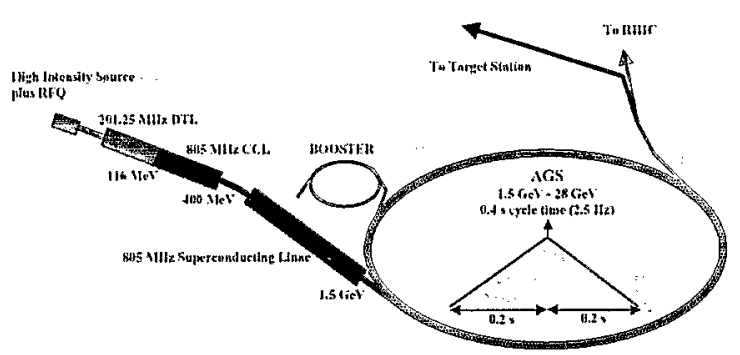

FGURE 1.: Schematic diagram of the accelerators for the "neutrino production".

\section{SUPERCONDUCTING LINAC (SCL).}

Two modifications are needed for the injector linac: (1) Upgrade the $200 \mathrm{MeV}$ linac to $400 \mathrm{MeV}$, based on the FERMILAB upgrade which was successfully completed in 1993 [4]; (2) Use the SNS high beta cryomodules [5] to $1.5 \mathrm{GeV}$ or higher energies in the 130 meter space: The Fermilab $200 \mathrm{MeV}$ linac was upgraded to $400 \mathrm{MeV}$ by replacing last four DTL tanks with seven $805 \mathrm{MHz}$ coupled cavity linac (CCL) modules [4]: These seven CCL modules. fit: within the existing linac tunnel enclosure, since the length of the CCL modules including the transition section is about 3 meters shorter than the last four. DTL tanks, Since 1993, the Fermilab linac has 
successfully accelerated a peak current of $50 \mathrm{~mA}$ with: pulse length of $50^{\prime \prime} \mu \mathrm{s}$ at repetition rate of $15 \mathrm{~Hz}$. The: average accelerating gradient in the CCL is $7.5 \mathrm{MV} / \mathrm{m}$; which is about four times higher than LAMPF at Los Alamos. The peak surface field is $37 \mathrm{MV} / \mathrm{m}$, which is 1.35 Kilpatrick. Each module has 4 sections and each: section has 16 cells. Each module is driven by a $12 \mathrm{MW}$. klystron: The focusing lattice is FODO with quadrupole gradient of approximately $20 \mathrm{~T} / \mathrm{m}$. The achieved sparking. rate at Fermilab is about $0.033 \%$ with $R F$ pulse length of $50 \mu$ s. [4]. Since our RF pulse length is about $1 \mathrm{~ms}$, the sparking rate could be higher. It would require some $R \&$ $\mathrm{D}$ efforts to minimize the sparking rate. In the Fermilab design nose corner is not.water-cooled. For our $1 \mathrm{~ms}$ long RF pulse length nose corner would require redesigning.

The SNS high beta module is 7.891 meters long. (including the warm section) and has 4 sections of 6 cells. The geometric beta for these modules is 0.81 . The designaccelerating field $\left(E_{0}\right)$ is about $22.8 \mathrm{MV} / \mathrm{m} \cdot\left(\mathrm{E}_{0} \mathrm{~T}=15.9\right.$ : $\mathrm{MV} / \mathrm{m}), 21$ modules will accelerate $\mathrm{H}^{-}$from $387 \mathrm{MeV}$ to $1300 \mathrm{MeV}$. At present; cavity testing at JLAB [6] shows. that the accelerating gradient of $30 \mathrm{MV} / \mathrm{m} \quad\left(\mathrm{E}_{0} \mathrm{~T}=21\right.$ $\mathrm{MV} / \mathrm{m}$ ) has been achieved. In 130 meter one can have 15 cryomodules. Assuming an accelerating gradient, $E_{0}=31$. $\mathrm{MV} / \mathrm{m}, 15$ cyromodules can accelerate $\mathrm{H}^{-}$from $400 \mathrm{MeV}$. to $1462 \mathrm{MeV}$. The energy can be upgraded to $1533 \mathrm{MeV}$ if the accelerating gradient $\left(E_{0}\right)$ of $33 \mathrm{MV} / \mathrm{m}$ (achieved at TESLA) becomes a reality in future for cavities with $\beta \leq 1$. Table II shows the general parameters for the linac.

The emittance: of the existing $200 \mathrm{MeV}$ linac is about $2 \pi \mathrm{mm}$ rad (rms, nor), which has to be reduced to 1. $\pi \mathrm{mm}$ rad to lower the losses during the injection process into the AGS [7].. The existing ion source and RFQ will be relocated next to. DTL tank 1 to meet the requirement [8]. The beam power at $1.5 \mathrm{GeV}$ is only $54 \mathrm{~kW}$, for hand on maintenance loss limit is watt $/ \mathrm{m}$, which translate to fractional loss limit of $3 \times 10^{-3}$. The estimated fractionali. loss is about $1 \times 10^{-4}$.

\section{Table II: Injector Linac parameters}

\begin{tabular}{ll}
\hline Kinetic Energy $(\mathrm{GeV})$ & 1.5 \\
Beam Power $(\mathrm{kW})$ & 54 \\
Average Beam Current $(\mu \mathrm{A})$ & 36 \\
Number of Proton per Bunch $\left(\times 10^{8}\right)$ & 8.70 \\
Repetition Rate $(\mathrm{Hz})$ & 2.5 \\
Beam Pulse Length $(\mu \mathrm{s})$ & 720 \\
Chopping Rate $(\%)$ & 65 \\
Emittance $(\pi$ mm mrad, nor) & 1.0 \\
Energy Spread $(\Delta \mathrm{E} / \mathrm{E}, 95 \%)$ & $\pm 0.001^{\prime} .$. \\
Energy Jitter $(\delta \mathrm{E}, \mathrm{MeV})$ & 2.5 \\
Drift Tube $\mathrm{Linac}(\mathrm{DTL})$ & \\
Energy $(\mathrm{MeV})$ & \\
Number of Tank & \\
Frequency $(\mathrm{MHz})$ & 116.5 \\
\hline
\end{tabular}

\begin{tabular}{|c|c|}
\hline \multicolumn{2}{|l|}{ Coupled Cavity Linac (CCL) } \\
\hline Energy (MeV) & 400 \\
\hline Frequency $(\mathrm{MHz})$ & 805.0 \\
\hline Number of Module' & 7 \\
\hline Number of Section per Module & 4 \\
\hline Number of Cells per Section. & 16 \\
\hline Bridge Coupler Length $(\beta \lambda)$ & $3 / 2$ \\
\hline Cavity Bore Radius $(\mathrm{cm})$ & 1.5 \\
\hline Accelerating Phase, $\phi_{\mathrm{s}}(\mathrm{deg})$ & -32 \\
\hline Average axial field, $\mathrm{E}_{0}(\mathrm{MV} / \mathrm{m})$ : & $7.1-8.1$ \\
\hline Kilpatrick . & 1.35 \\
\hline RF Power per module (MW) & $<12$ \\
\hline Transverse Focusing & FODO \\
\hline Average Transverse Phase Advance (deg) & 79 \\
\hline Quadrupole Bore Radius (cm) & 2.0 \\
\hline Quadrupole Magnetic Length $(\mathrm{cm})$ & $8: 0$ \\
\hline Quadrupole Pole Tip Field (kG). & 4.6 \\
\hline \multicolumn{2}{|l|}{ Superconducting Linac (SCL) . } \\
\hline Energy $(\mathrm{GeV})$ & 1.46 \\
\hline Frequency $(\mathrm{MHz})$ & 805.0 \\
\hline Number of Cryomodule & 15 \\
\hline Number of Cavity per Cryomodule & 4. \\
\hline Number of Cell per Cavity & 6 \\
\hline Geometric Beta & 0.81 \\
\hline Slot Length (m) & 7.891 \\
\hline Warm Insertion Length (m) & 1.6 \\
\hline Average Axial Field, $\mathrm{E}_{0}(\mathrm{MV} / \mathrm{m})$ & 31 \\
\hline Peak Surface Field, $E_{p}(M V / m)$ & 51.2 \\
\hline Accelerating Phase, $\phi_{\mathrm{s}}(\mathrm{deg})$ & -19.5 \\
\hline Number of Cavities per Klystron „.. & 1 \\
\hline Klystron Power $(\mathrm{kW})$ & 550 \\
\hline Temperature $\left({ }^{\circ} \mathrm{K}\right)$ & 2.1 \\
\hline Transverse Focusing & FODO \\
\hline Transverse Phase Advance (deg) & 70 \\
\hline Quadrupole Length $(\mathrm{cm})$. & 40 \\
\hline Quadrupole bore Radius (cm) & 4.0 \\
\hline
\end{tabular}

\section{AGS UPGRADE}

In its current operation, the AGS receives four batches of $1.5 \mathrm{GeV}$ proton beam from the Booster.synchrotron in about 0.5 second. The typical intensity achieved for slow extracted beam operation is about $70 \times 10 \ldots$ In the proposed AGS upgrade for the neutrino beam program, a new $1.5 \% \mathrm{GeV}$ superconducting linac will be used as injector which can provide $89 \times 10^{12}$ proton with injection time of less than $1 \mathrm{~ms}$. To provide $1 \mathrm{MW}$ beam power for neutrino production, the AGS has to be cycled at $2.5 \mathrm{~Hz}$, instead of $0.5 \mathrm{~Hz}$. For this improved capability, several 
major upgrades of the AGS have to be implemented: (1) the new direct injection from the SCL with $\mathrm{H}_{\text {i stripping }}$ foil system;:! (2) the new main magnet power supply and:: its six-loop configuration for the powering of the latticemagnets; (3) the new $\mathrm{RF}$. accelerating cavity and its associated power switching system for doubling the accelerating voltage operated at $2.5 \mathrm{~Hz}$; (4) the new single turn fast extraction system for beam delivery to the target; : (5) the new collimation and radiation shielding system to keep the beam losses at an acceptable level.

\section{BEAM TRANSFER LINE}

The beam is transported $\sim 190 \mathrm{~m}$ from the AGS to the U-line spur using present RHIC transfer line magnets. Thei: new beam transport begins at this point. To direct the beam toward the. Homestake mine in South Dakota, the: beam must be bent 68 degrees; 4 seconds to the west of the U-line direction and 11:26 degrees downward. BNL is located on an aquifer that is the sole-source for Suffolk: county drinking: water. A beam layout has been developed: that takes the beam up and over a 42 -meter (beam height) hill to the production target and decay channel. This keeps the target; decay channel and beam dump at or above the present ground level and well above the Long Island: water table. Figure 2 shows a 3-dimensional view of this beam transfer line. A beam layout was . developed to incorporate separate vertical and horizontal bends. The 17-degree vertical bend up begins near the end of the present U-line tunnel about 80 meters from the beginning; : of the new transport. The 80-meter drift before beginning. the vertical bend up is necessary to avoid conflicts with the RHIC transfer line utilities and entrance labyrinths: This bend is followed by a 46-meter drift to allow the beam to reach a height sufficient to allow, after a 68 . degree horizontal bend and a vertical downward bend, a 200-meter decay tunnel: (target to dump) down the hill to a dump, keeping the front face of the dump at ground level,: In order to come out of the horizontal and final; vertical: bend with the beam heading toward Homestake the actual: bend angles had to be adjusted to account for the fact that the bends are accomplished on the hill. The "ground. level" horizontal and vertical angles, relative to the U-line . beam direction, that point the beam toward Homestake are 68 and 11.3 degrees respectively. The required horizontal: bend is .72 .1 degrees followed by a 17.2 vertical bend down in the $y-z$ plane defined by the beam as it exits the horizontal bend.

\section{TARGET STATION-AND NEUTRINO BEAM}

To achieve the $1 \mathrm{MW}$ upgrade option of the proton driver at BNL, serious consideration must be given to the target selection. In evaluating the various choices of target materials and of target/horn configurations, the following ... concerns are being addressed: : .

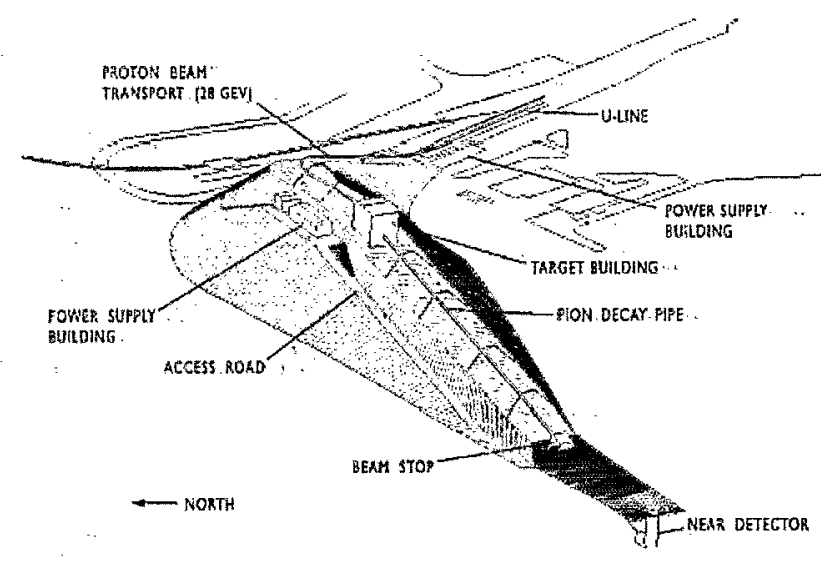

Figure 2: Elevation view of the neutrino beam line to Homestake, South Dakota.

- Optimization of neutrino flux;

- Heat removal from the target and horn,

- Survivability of the target intercepting energetic, high intensity proton bunches,

- Irradiätion and integration issues.

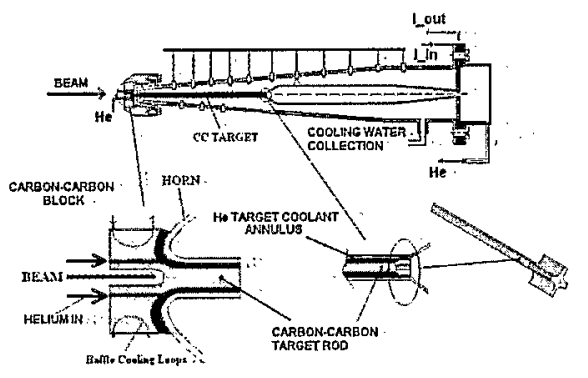

Figure 3: Proposed graphite target and horn configuration

The design of the target/horn configuration is shown in Fig. 3.The material selected for the superbeam experiment is a Carbon-Carbon composite. It is 3-D weaved material and exhibits extremely low thermal expansion for the temperatures up to $1000^{\circ} \mathrm{C}$ while for the higher. temperatures it responds like graphite. This property is significant in the sense that the thermo elastic stresses. induced by intercepting the beam will be quite small thus extending the life of the target.

In the current option the target is an 80-cm long cylindrical rod with $12 \mathrm{~mm}$ diameter sizes. The $12 \mathrm{~mm}$ diameter target is chosen to intercept $100 \mathrm{TP}, 2 \mathrm{~mm} \mathrm{rms}$ proton beam. With this beam size, the total energy. deposited as heat in the target is $7.3 \mathrm{~kJ}$ with peak. temperature rise of about $280^{\circ} \mathrm{C}$. Heat will be removed from the target through forced convection of helium through the outside surface

Using detailed finite element analysis incorporating the entire heat transfer scheme and the transient nature of the two inputs (protons and current) the "steady-state". temperatures in the target and horn were calculated and shown in Figures 4 and 5. 


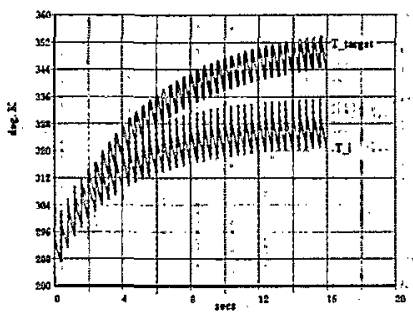

Figure 4: Transient temperatures in the horn conductor

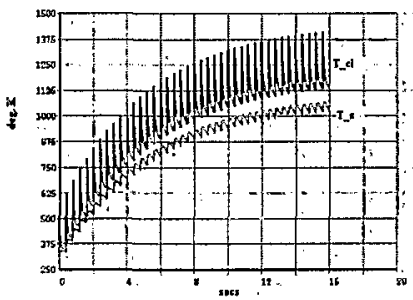

Figure 5: Transient temperatures in the C-C target

The resultant $v_{\mu}$ spectrum is shown in Fig. 6. Which is. used to study various neutrino processes and event rates at. distant detectors.

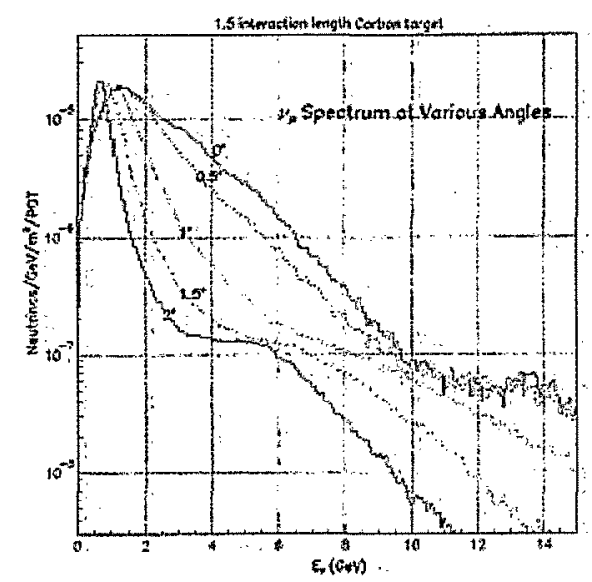

Figure 6: Wide band horn focused muon neutrino spectrum for $28 \mathrm{GeV}$ protons on a Carbon target. Spectrum of neutrinos is calculated at various angles with , $x$... respect to the $200 \mathrm{~m}$ decay tunnel axis at the AGS and at a distance of $1: \mathrm{km}$ from the target.

\section{BEAM DYNAMICS ISSUES}

For $1 \mathrm{MW}$ super neutrino beam facility; the AGS has new injection scheme and 10 times more proton per second then current AGS operations, these results in new beam : : dynamics issues to consider. These issues are; (1). injection painting, (2) transition crossing, (3) ring impedances; and (4) magnetic multipoles generated by eddy current due to higher rape rage in the AGS magnets:

$1.5 \mathrm{GeV} \cdot \mathrm{H}^{-}$will be injected through $300 \mu \mathrm{g} / \mathrm{cm}^{2}$. stripper carbon foil into the AGS for: 240 turns. Beam loss: in the injection process is one of the most important issues: for a high power proton: accelerator: For super neutrino beam operation following are the relevant: power and beam loss estimate during the injection: (1) $54 \mathrm{~kW}$ injected beam power at $1.5 \mathrm{GeV}, 0.90 \mathrm{~kW} \mathrm{H}$ ions missing the foil, (3) $0.90-5.4 \mathrm{~kW} \cdot \mathrm{H}^{0}$ from stripping foil (This rate depends on the foil thickness of the stripping foil), and (4).54 W stripped electron. An optimum,thickness and size of the injection foil is needed to balance these losses against aforementioned $\mathrm{H}^{-}$and $\mathrm{H}^{0}$ losses. There are additional beam losses due to (a) nuclear scattering, (b) multiple scattering, and (c) energy loss and struggling as some part of the circulating proton traverse through the foil. To reduce these looses an. optimization of the collapse time of the injection bump magnet is needed.

A direct effect of linac beam emittance is the halo/tail generation in the circulating beam [8]: Figure 7 shows the estimated halo/tail generation in the beam as a function of normalized RMS emittance of linac: beam. Here; the Halo/tail generation is defined as the ratio of number of particles ... with emittance larger than the designed acceptance of $49 \pi \mathrm{mm}$-mrad to the total number of particles in the circulating beam. The existing ion source and RFQ has to be relocated next to DTL tank 1 to meet emittance requirement for the. AGS injection with low loss.

TABLE 3 Simulation parameters.

\begin{tabular}{ll}
\hline Horizontal beta at the injection: & $28.0 \mathrm{~m}$ \\
Vertical beta at the injection : & $8.0 \mathrm{~m}$ \\
Horizontal emittance of injected beam & $2 \pi \mathrm{mm}$-mrad \\
Vertical emittance of injected beam & $2 \pi \mathrm{mm}-\mathrm{mrad}$ \\
Horizontal beam size at injection, $\sigma_{\mathrm{x}}$ & $5.2293 \mathrm{~mm}$ \\
Vertical beam size at injection, $\sigma_{\mathrm{y}}$ & $2.7952 \mathrm{~mm}$ \\
Horizontal Foil size $\left(2.5 \sigma_{\mathrm{x}}\right)$ & $13.0731 \mathrm{~mm}$ \\
Vertical foil size $\left(2: 5 \sigma_{\mathrm{y}}\right)$ & $6.9878 \mathrm{~mm}$ \\
\hline
\end{tabular}

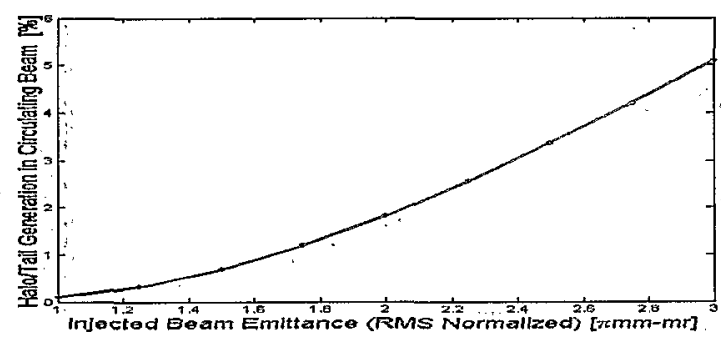

FIGURE 7:: The estimated halo/tail generation in the circulating beam: as functions of normalized RMS emittance of injected beam.:

For longitudinal painting, simulations shows that a relatively low rf voltage of $450 \mathrm{kV}$ at injection is necessary to limit the beam momentum spread to about $0.5 \%$ and longitudinal emittance of 0.8 to eVs per bunch and the chopping rate $0.65 \ldots$ Such a small longitudinal emittance is important to limit beam losses during the transition crossing.in the AGS.

The proton beam crosses the transition energy at $\gamma_{\mathrm{T}}=8.5$. During a non- adiabatic time $\pm \mathrm{T}_{\mathrm{c}, \mathrm{t}}$ the beam may experience emittance growth and beam loss caused by: chromatic non-linear mismatch, beam self-field mismatch 
and beam instabilities. It is necessary to use the transition jump method to effectively increase the rate of transition crossing. The required amount " of : transition . jump.. $\Delta \gamma_{\mathrm{T}}= \pm 0.5$ during a time of $1 \mathrm{~ms}$ or shorter. The expected beam loss is about $0.2 \%$ for a $0.8 \mathrm{eVs}$ longitudinal beam emittance. While the condition for.: machine hands-on maintenance of average beam loss of $1 \mathrm{~W} / \mathrm{m}$ corresponds: to a fractional uncontrolled beam loss of $0.3 \%[9]$ :

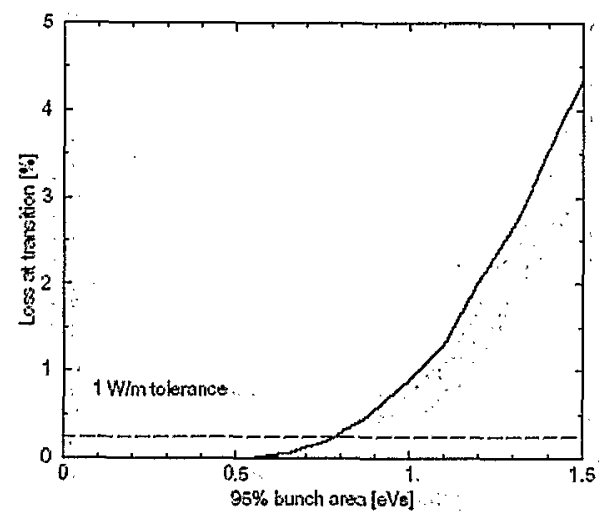

Figure 8: Expected fractional beam loss during transition .. as a function of the initial (95\%) longitudinal beam area obtain with computer code TIBETAN:

The beam instability: considered for the super neutrino beam operation for the AGS at high energy are; (a) longitudinal instability around transition, and (b) the transverse instability: above transition. The longitudinal impedance needs to be less than $12 \Omega$ to avoid longitudinal microwave instabilities. The measured AGS longitudinal impedance is about $30 \Omega$. All bellows in the AGS (about 450) are unshielded. The "chamber: steps, incliding the connection from dipole to quadrupole and the BPM housing, are not tapered. With limited effort of: shielding and tapering, the AGS impedance can be reduce to about. $12 \Omega$ : The longitudinal space charge impedance is about $10 \Omega$ at transition, which is capacitive, has the effect of canceling the indirctive broadband:impedance. In summary, since the required intensity of $8.9 \times 10^{13}$ is only. marginally higher than the current intensity of $7.3 \times 10^{13}$ : The beam instability: during acceleration and transition: crossing can be avoided.

Presently the magnet cycle of the AGS accelerator has a period of $\sim 3.5 \mathrm{~s}$ with rise time of $200 \mathrm{~ms}$ between : the injection energy and top energy. The proposed magnet cycle of $2.5 \mathrm{~Hz}$ : for "the neutrino: production operation: will reduce the time : between the injection and extraction to $\sim 90 \mathrm{~ms}$. The time varying magnetic flux generated by the excitation of the main magnet generates eddy currents in the wall of the vacuum chamber of the circulating beam. The eddy currents generated on the wall of the vacuum chamber have the following adverse effect: (a) ohmic heating on the wall of the vacuum chamber; (b.) introduce magnetic multipoles including dipole field. Experimental measurements of the temperature rise of the vacuum chamber of the AGS have been performed for a single AGS c-type magnet when the coil of the magnet is subject to time varying sinusoidal current and found the rise in temperature is acceptable. Calculations " shows that the magnetic multi-pole generated due to the eddy current are low enough not to cause beam instability:

\section{CONCLUSIONS}

We have produced a design for 1 . MW AGS-based neutrino superbeam facility which "can be further upgraded to $4 \mathrm{MW}$ by combination of the following. improvements 1), increase the AGSintensity to $1.8 \times 10^{14}$ $\mathrm{ppp}$, and 2) increase the AGS rep rate to $5.0 \mathrm{~Hz}, 3$ ) raise the proton beam energy to $40 \mathrm{GeV}$ and 4 ) improve on the horn focusing, at the target. The associated problem in beam dynamics, power supply, rf system, beam losses and radiation protection are under study and shown to be feasible if such a capability is required by the physics experiments

Several $R \& D$ programs in the design of the superconducting cavity and the irradiation testing of target materials are actively pursued to improve on the design.:

\section{REFERENCES}

[1]"Very Long Baseline Neutrino Oscillation Experiment for Precise Measurements of Mixing Parameters and: $C P$. Violating Effects", M. V. Diwan; et al., PRD 68 (2003).

[2] "Report of the BNL. Neutrino. Working Group"; M. Diwan, W: Marciano and W. Weng, Informal :Report, BNL-69395; October, 2002:

[3] "The: AGS-Based Super Neutrino Beam: Facility, Conceptual : Design Report", Editors: W. T. Weng, $M$. Diwan, and D. Raparia, Informal Report, BNL-732102004-IR, October, 2004.

[4] "The Commissioning and initial operation of the Fermilab 400 MeV : linac", E. S. McCrory, 1994 International, Linac Conference, Tsukuba; Japan, August 1994.

[5] "The SNS Superconducting Linac System"; C. Rode, et al., 2001 Particle Accelerator Conference, Chicago, pp 619-623, 2001:

[6] C. Rode, Talk given in SNS.DOE review May 2004:

[7] "Effect of Halo on the AGS Injection from $1: 2, \mathrm{GeV}$. Linac"; W. T. Wang et al., AIP conference Proceeding. 693, pp85, 2003.

[8] "Proposal to Reduce Tränsverse Emittance for BNL $200 \mathrm{MeV}$ Linac", D: Raparia, to be published in 2004 linac conference.

[9]." Transition Crossing for the BNL Super Neutrino Beam ":, J. Weil and N. Tsoupas, 2004 European Particle Accelerator Conference, Lucerne, July,: 2004. 